\title{
Leishmaniose visceral: características clínico-epidemiológicas em crianças de área endêmica
}

\author{
Visceral leishmaniasis: clinical and epidemiological features of children in an endemic area \\ Márcia J. A. Queiroz ${ }^{1}$, João G. B. Alves ${ }^{2}$, Jailson B. Correia ${ }^{3}$
}

\section{Resumo}

Objetivo: Descrever as características clínico-epidemiológicas, o tratamento e a letalidade das crianças internadas com leishmaniose visceral em um hospital pediátrico de referência.

Métodos: Análise retrospectiva dos dados biológicos, demográficos, clínicos e laboratoriais das crianças internadas no Instituto Materno-Infantil de Pernambuco, em Recife, no período compreendido entre 1996 e 2001.

Resultados: Foram incluídas 431 crianças, de 4 meses a 13,7 anos de idade, sendo que $50,3 \%$ eram do sexo feminino, e $82,5 \%$ eram do interior do estado de Pernambuco. Cerca de $70 \%$ dos domicílios eram de alvenaria, $70 \%$ não dispunham de água encanada ou sistema de esgoto sanitário, e o tempo médio de permanência das mães na escola foi de 3 anos. Esplenomegalia e febre estavam presentes em $97 \%$ e $95,6 \%$ dos casos, respectivamente, e $44,5 \%$ dos pacientes eram subnutridos. Em 47 (10,9\%) dos pacientes foi detectada infecção na admissão. O nível médio de hemoglobina foi de $6 \mathrm{~g} / \mathrm{dl}$, de leucócitos $3.516 / \mathrm{mm}^{3}$ e de plaquetas $118.641 / \mathrm{mm}^{3}$. O tratamento de escolha foi o glucantime ( $98 \%$ dos casos), e em sete pacientes, a anfotericina $B$ foi utilizada. A letalidade foi de $10,2 \%$, sendo que as principais causas imediatas de óbito foram atribuídas a infecções associadas, hemorragias e insuficiência hepática.

Conclusões: Os autores destacam as características clínicas, epidemiológicas e laboratoriais da leishmaniose visceral em área endêmica, além do diagnóstico tardio e alta letalidade, sugerindo a capacitação de profissionais de saúde para o reconhecimento precoce e tratamento adequado da doença e suas complicações.

J Pediatr (Rio J). 2004;80(2):141-6: Leishmaniose visceral, calazar, infância, diagnóstico, tratamento, letalidade.

\section{Introdução}

Segundo a Organização Mundial de Saúde (OMS), as leishmanioses afetam cerca de dois milhões de pessoas por ano, com 500 mil casos da forma visceral. Estima-se que

1. Mestre em Saúde Materno-Infantil pelo Instituto Materno-Infantil de Pernambuco (IMIP). Médica do IMIP.

2. Doutor em Medicina pela Universidade Federal de Pernambuco. Professor adjunto da Universidade de Pernambuco. Médico do IMIP.

3. Doutorando em Medicina pela Universidade de Liverpool. Professor auxiliar da Universidade de Pernambuco. Médico do IMIP.

Artigo submetido em 28.04.03, aceito em 26.11.03.

\section{Abstract}

Objective: To describe the clinical and epidemiological features of children with visceral leishmaniasis admitted to a pediatric referral hospital, and to describe treatment measures and the case fatality rate.

Methods: Retrospective analysis of biological, demographic, clinical and laboratory data from children with visceral leishmaniasis admitted to Instituto Materno Infantil de Pernambuco (Recife, state of Pernambuco, northeastern Brazil) between 1996 and 2001.

Results: 431 children were included in the study. Age ranged from 4 months to 13.7 years. $50.3 \%$ were female and $82.5 \%$ came from the interior of the state of Pernambuco. $70 \%$ of the patients lived in brick homes, and $70 \%$ were not served with piped water and sewage services. Average maternal schooling was 3 years. Clinical presentation included splenomegaly $(97 \%)$, fever $(95.6 \%)$ and malnourishment $(44.5 \%)$. Associated infections were diagnosed in $10.9 \%$ of cases. The mean values for laboratory variables were:

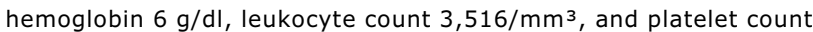
$118,641 / \mathrm{mm}^{3}$. The first line treatment used in $98 \%$ of the cases was glucantime. Amphotericin B was used in seven cases. The case fatality rate was $10.2 \%$. The main immediate causes of death were associated infections, bleeding and liver failure.

Conclusions: Health care workers should be trained for the early recognition and appropriate management of visceral leishmaniasis and its complications.

J Pediatr (Rio J). 2004;80(2):141-6: Visceral leishmaniasis, kalaazar, childhood, diagnosis, treatment, case fatality rate.
350 milhões de pessoas estão expostas ao risco de infecção, com uma prevalência de 12 milhões de infectados ${ }^{1,2}$.

A leishmaniose visceral ou calazar está amplamente difundida no Brasil, com casos autóctones notificados em pelo menos 19 estados da federação, distribuídos em quatro das cinco regiões, permanecendo indene apenas o Sul ${ }^{3}$. Entre 1984 e 2000, foram notificados 67.231 casos $^{4}$. A Região Nordeste concentra mais de $90 \%$ das notificações, com registros de casos em todas as suas unidades federadas. Em Pernambuco ${ }^{5}$, foram notificados 1.203 casos no 
período de 1990 a 1997. Nas últimas décadas, o estado tem mostrado forte tendência à urbanização, com a ocorrência de surtos epidêmicos em várias capitais ${ }^{6}$ constituindo um sério problema de saúde pública que ameaça a população e preocupa as autoridades sanitárias.

A leishmaniose visceral (LV) é uma infecção sistêmica causada por um protozoário do gênero Leishmania. No Brasil, o principal vetor é o Lutzomya longipalpis, sendo o cão doméstico o reservatório mais importante e o homem o hospedeiro final ${ }^{3}$. A maioria dos casos é de infecção assintomática ou que desenvolvem sintomas moderados ou transitórios como diarréia, tosse seca, adinamia, febrícula, sudorese e discreta hepatoesplenomegalia ${ }^{7}$, que podem evoluir ou não para a forma clássica da doença. O quadro clássico consiste de febre, hepatoesplenomegalia, com esplenomegalia volumosa, perda de peso, tosse, diarréia, dor e distensão abdominal. Icterícia e envolvimento renal têm sido descritos. Na fase mais tardia da doença, os pacientes podem desenvolver edema e ascite ${ }^{1}$.

O diagnóstico baseia-se no encontro do parasita em tecido de medula óssea, baço, fígado ou linfonodos ${ }^{7}$. Foram desenvolvidos vários testes sorológicos para o diagnóstico (exemplos: fixação do complemento, imunofluorescência indireta, teste de aglutinação direta, ELISA e Dot-ELISA), assim como técnicas de biologia molecular (reação em cadeia da polimerase), porém persistem alguns problemas quanto à sensibilidade, especificidade, disponibilidade e custo desses testes na prática clínica ${ }^{8}$. Quando não há possibilidade de diagnóstico laboratorial, o início do tratamento é baseado nos achados clínico-epidemiológicos ${ }^{9}$.

Os antimoniais pentavalentes continuam sendo a droga de primeira escolha para o tratamento, e a anfotericina B é a droga de segunda linha, em casos de resistência ao antimoniato ${ }^{3}$. Recentemente, uma nova droga de administração oral, a miltefosina, tem-se mostrado efetiva no tratamento da LV na Índia ${ }^{10}$. Infecções, hemorragias e anemia grave são responsáveis pela maioria das mortes, e o retardo no diagnóstico, a baixa idade e a desnutrição são implicados como importantes fatores que contribuem para o óbito 3,7 .

Os autores descreveram os dados epidemiológicos, clínicos e laboratoriais dos pacientes internados com calazar em um hospital pediátrico de referência, destacando a importância do diagnóstico e tratamento precoces para evitar a alta letalidade.

\section{Casuística e métodos}

Foram internados 445 pacientes de até 14 anos de idade com LV no Instituto Materno-Infantil de Pernambuco (IMIP), um dos hospitais de referência do estado de Pernambuco para diagnóstico e tratamento da doença, no período de maio de 1996 a dezembro de 2001. Oito prontuários não foram encontrados e outros foram excluídos porque, apesar do diagnóstico final constar como calazar, não preencheram os critérios de inclusão da pesquisa. Obtivemos, então, 431 prontuários. Os dados foram obtidos pela análise retrospectiva dos prontuários de crianças admitidas com LV, através de um formulário padronizado, desenvolvido pelos pesquisadores, onde constavam: identificação, procedência, características do domicílio, escolaridade materna, queixas principais e tempo de duração, história de tratamento prévio, exame físico, exames laboratoriais e tratamento. Foram incluídos todos os pacientes com diagnóstico comprovado através de mielograma, teste de aglutinação direta $($ DAT) $\geq 1 / 1.600$ ou imunofluorescência indireta (IFI) $\geq 1 / 40$ e também aqueles casos prováveis, com o mielograma negativo, mas suspeita clínico-epidemiológica e laboratorial (pancitopenia) de calazar, excluídas outras patologias.

O grau de nutrição foi avaliado a partir do índice de peso por idade, no momento da admissão, considerando como padrão de referência a curva do National Center of Health Statistics dos Estados Unidos (NCHS). Crianças com edema envolvendo, no mínimo, os pés, foram considerados gravemente desnutridos (desnutrição edematosa). Pancitopenia foi considerada quando se encontrou leucopenia (contagem de leucócitos $\leq 5.000 / \mathrm{mm}^{3}$ ), anemia (nível de hemoglobina $<11 \mathrm{~g} / \mathrm{dl}$ entre 7 meses e 5 anos, $<11,5 \mathrm{~g} / \mathrm{dl}$ entre 6 e 9 anos, $<12 \mathrm{~g} / \mathrm{dl}$ em adolescentes do sexo feminino e $<12,5$ $\mathrm{g} / \mathrm{dl}$ em adolescentes do sexo masculino) e plaquetopenia (contagem de plaquetas $<150.000 / \mathrm{mm}^{3}$ ).

A análise foi realizada no programa Epi-Info $6.04 \mathrm{~b}$ para a distribuição de freqüência, média e desvio padrão dos dados obtidos, quando necessário.

O estudo obteve a aprovação do Comissão de Ética do Hospital em relação ao seu aspecto ético e científico antes do início da coleta de dados.

\section{Resultados}

A distribuição dos casos ao longo dos anos está apresentada na Tabela 1. O maior número de casos (180) ocorreu no ano de 2000 , porém a maior letalidade foi observada em $1999(14,8 \%)$.

Em relação ao sexo, 50,3\% das crianças eram do sexo feminino. A idade média foi de 4,2 anos ( $D P= \pm 3$ ), e a criança mais nova tinha 4 meses. A faixa etária mais acometida foi a de menores de 5 anos ( $68,2 \%$ dos casos),

Tabela 1 - Distribuição anual das crianças internadas por calazar no IMIP, no período de maio de 1996 a dezembro de 2001

\begin{tabular}{lccc}
\hline Ano & Casos & $\begin{array}{c}\text { Óbitos } \\
\mathbf{N}\end{array}$ & $\%$ \\
\hline 1996 & 25 & - & - \\
1997 & 34 & 2 & 5,9 \\
1998 & 26 & 2 & 7,2 \\
1999 & 94 & 14 & 14,8 \\
2000 & 180 & 18 & 10,0 \\
2001 & 72 & 08 & 11,1 \\
Total & 431 & 44 & 10,2 \\
\hline
\end{tabular}

Fonte: Pesquisa HGP/IMIP. 
e $9 \%$ das crianças internadas eram menores de 1 ano. Dos 317 pacientes em que foi possível obter a informação sobre escolaridade materna, constatou-se que o tempo médio de permanência na escola foi de 3,1 anos ( $D P= \pm 3$ ). Dentre as características do domicílio, foi possível obter a informação sobre a existência ou não de água e sistema de esgoto sanitário em 358 prontuários, e cerca de $70 \%$ das casas não dispunham desses serviços.

A maioria dos pacientes procedia do estado de Pernambuco ( $99 \%)$, três eram de Alagoas e um da Paraíba. Do total das crianças, $82,5 \%$ concentravam sua origem no interior de Pernambuco e $14,8 \%$ eram da região metropolitana do Recife.

Em relação à situação nutricional, verificou-se uma freqüência de subnutrição na população estudada de 44,5\%, enquanto $26,9 \%$ de todas as crianças eram desnutridas graves.

A duração da doença desde o início dos sintomas até a hospitalização variou de 2 a 365 dias, com média de 42,7 dias ( $D P= \pm 45$ ), sendo que $88,7 \%$ dos casos arrastaramse com os sintomas por período menor que 60 dias. 0 tempo médio de internamento foi de 11,2 dias ( $D P= \pm 5,7$ ), variando de 0 a 47 dias. Entre os sintomas referidos pelos familiares, ressaltam-se a febre, o aumento do volume abdominal, a palidez, a anorexia e a tosse como os mais freqüentes (Tabela 2). Aproximadamente $50 \%$ das mães utilizaram algum tipo de medicação, principalmente antibiótico. Houve relato de uso prévio de tratamento específico para calazar por quinze genitoras.

Tabela 2 - Principais sintomas e achados clínicos das crianças internadas com calazar no IMIP, no período de maio de 1996 a dezembro de 2001

\begin{tabular}{lcc}
\hline Sintomas & $\mathbf{N}$ & $\%$ \\
\hline Febre & 412 & 95,6 \\
Aumento do abdômen & 277 & 64,3 \\
Palidez & 250 & 58,0 \\
Anorexia & 215 & 49,9 \\
Tosse & 181 & 42,0 \\
Emagrecimento & 163 & 37,8 \\
Astenia & 163 & 37,8 \\
Dor abdominal & 73 & 16,9 \\
Diarréia & 55 & 12,8 \\
Edema & 71 & 16,5 \\
Infecção & 50 & 11,6 \\
& & \\
\hline Achados clínicos & $\mathbf{N}$ & $\%$ \\
\hline Esplenomegalia & 418 & 97,0 \\
Palidez & 393 & 91,2 \\
Hepatomegalia & 376 & 87,2 \\
Subnutrição & 191 & 44,5 \\
Edema & 69 & 16,0 \\
Dispnéia & 53 & 12,2 \\
Infecção & 47 & 10,9 \\
Hemorragia & 49 & 11,4 \\
Icterícia & 19 & 4,4 \\
\hline & & \\
\hline & & \\
\hline
\end{tabular}

Fonte: Pesquisa HGP/IMIP.
Também na Tabela 2 estão apresentados os achados do exame físico na admissão, registrando-se esplenomegalia, palidez e hepatomegalia como os mais freqüentes. Em 47 $(10,9 \%)$ das crianças foi detectado algum tipo de infecção na admissão, sendo as mais freqüentes: pneumonia em $66 \%$ dos casos, otite em $18,4 \%$ dos casos e sepse em $8,2 \%$ dos casos.

Os dados hematológicos referentes à série vermelha, branca e plaquetária no momento da admissão são mostrados na Tabela 3. O nível médio de hemoglobina foi de $6 \mathrm{~g} / \mathrm{dl}$, sendo necessárias transfusões de hemácias em 170 pacientes durante o internamento. A contagem média de leucócitos foi de $3.516 / \mathrm{mm}^{3}$, e o número de leucócitos foi inferior a $5.000 / \mathrm{mm}^{3}$ em 367 casos (85,3\%). A contagem média de neutrófilos foi de $1.215 / \mathrm{mm}^{3}$, e evidenciaram-se valores inferiores a $500 / \mathrm{mm}^{3}$ em $15,9 \%$ pacientes. A contagem média de plaquetas foi de $118.641 / \mathrm{mm}^{3}$, sendo que 0 número de plaquetas no período pré-tratamento foi $\leq 150.000 / \mathrm{mm}^{3}$ em $68,4 \%$ dos casos; foram necessárias 43 transfusões de plaquetas.

Tabela 3 - Dados hematológicos das crianças internadas com calazar no IMIP, no período de maio de 1996 a dezembro de 2001

\begin{tabular}{lccc}
\hline Parâmetros & Média \pm DP & $\begin{array}{c}\text { Valor } \\
\text { Mínimo }\end{array}$ & Máximo \\
\hline Hemoglobina $(\mathrm{g} / \mathrm{dl})$ & $6,1 \pm 1,7$ & 1,8 & 11,2 \\
Leucócitos $\left(\mathrm{mm}^{3}\right)$ & $3.516 \pm 1.923$ & 600 & 16.300 \\
Neutrófilos $\left(\mathrm{mm}^{3}\right)$ & $1.215 \pm 1.052$ & 32 & 9.682 \\
Plaquetas $\left(\mathrm{mm}^{3}\right)$ & $118.641 \pm 88.359$ & 2.540 & 808.000
\end{tabular}

Fonte: Pesquisa HGP/IMIP.

A confirmação do diagnóstico foi possível em 79,4\% dos casos. A pesquisa do parasita pelo mielograma foi realizada em 412 pacientes, sendo positiva em 311 (75,5\%) dos casos. O DAT foi positivo em 28 , e a IFI em três pacientes, respectivamente. Nos casos em que não foi possível confirmar o diagnóstico por esses métodos, a análise dos dados epidemiológicos, clínicos e laboratoriais foi suficiente para a instituição da terapêutica.

O tratamento de escolha para a leishmaniose visceral foi o glucantime em $98 \%$ dos pacientes. Sete desses pacientes utilizaram a associação glucantime e alopurinol. Sete pacientes permaneceram sintomáticos após o primeiro esquema utilizado e foram tratados com anfotericina B. O tempo médio de regressão da febre foi de 3 dias ( $D P= \pm 2,7$ ), e 50 pacientes não apresentaram febre durante o internamento. Sete pacientes foram a óbito antes de iniciar medicação específica para a doença.

Nos 44 óbitos registrados, o tempo médio de internamento foi de 10 dias ( $D P= \pm 9$ ), e dois pacientes morreram no dia do internamento. Dentre as causas imediatas de 
óbito, infecção esteve presente em 32 casos $(72,7 \%)$, hemorragia em $26(59,0 \%)$ e insuficiência hepática em 14 $(31,8 \%)$, sendo que $70 \%$ dos pacientes tinham mais de um agravo associado (Tabela 4). As infecções mais freqüentes foram pneumonia em $26(66,7 \%)$ e sepse em $15(38,5 \%)$ pacientes. Convulsão e insuficiência respiratória foram observadas cada uma em nove pacientes no momento que antecedeu o óbito.

Tabela 4 - Principais causas imediatas de óbito em crianças internadas por calazar no IMIP, no período de maio de 1996 a dezembro de 2001

\begin{tabular}{lcc}
\hline \multirow{2}{*}{ Causas } & \multicolumn{2}{c}{ óbito } \\
& N & \% \\
\hline \multirow{2}{*}{ Infecções associadas } & 32 & 72,7 \\
Hemorragia & 26 & 59,0 \\
Insuficiência hepática & 14 & 31,8 \\
Hemorragia + infecção & 10 & 22,7 \\
Hemorragia + infecção + insuficiência hepática & 10 & 22,7 \\
Anemia grave & 8 & 18,2 \\
Infeç̧ão + insuficiência hepática & 4 & 9,0 \\
\hline
\end{tabular}

Fonte: Pesquisa HGP/IMIP

\section{Discussão}

Uma importante característica da leishmaniose visceral é que, quanto maior a incidência da doença, maior o risco para as crianças mais jovens, fato já documentado no Brasil, onde a preferência da doença pela população infantil vem se mantendo ao longo dos anos $3,5,7,11$. Essa característica é semelhante ao observado no presente estudo, no qual a LV predominou nos primeiros 5 anos de vida, faixa etária de $68,2 \%$ dos doentes. Uma vez que a imunidade duradoura se desenvolve com a idade ${ }^{1}$, é provável que a maior incidência de doença e de óbito no grupo de menor idade dependa da maior suscetibilidade à infecção e da depressão da imunidade observada nesta faixa etária.

A literatura aponta o sexo masculino como mais susceptível ao adoecimento ${ }^{3,5,12}$. Nesta série, crianças do sexo masculino e feminino foram igualmente afetadas. Ressaltamos que o problema da maior prevalência da doença entre as pessoas do sexo masculino ainda não está totalmente esclarecido, postulando-se a existência de um fator hormonal ligado ao sexo ou à exposição ${ }^{13}$.

A distribuição da doença no Brasil mostra uma tendência cíclica, com o pico observado no ano 2000, refletido em nossa casuística. $O$ aumento do número de casos durante 0 período estudado decorre, provavelmente, da expansão das áreas endêmicas, levando ao aparecimento da doença na periferia das grandes cidades, onde não era conhecida até aquele momento, dificultando o diagnóstico e levando a um aumento da letalidade. De fato, desde meados dos anos 80 , o calazar vem claramente se expandindo para regiões rurais indenes e para a periferia de alguns centros urbanos ${ }^{3,14}$. Em Pernambuco, a LV, que se encontrava basicamente no litoral e alto sertão, atualmente atinge quase todo o estado ${ }^{15}$, inclusive a região metropolitana do Recife ${ }^{16}$, fato destacado nesta pesquisa, na qual quase $15 \%$ das crianças internadas eram provenientes desta região.

A precariedade das condições socioeconômicas está associada à maior incidência de várias doenças infecciosas, entre elas a LV, como se observa nesta casuística, em que, apesar da maioria dos domicílios serem de alvenaria, a disponibilidade de água encanada e esgoto sanitário ainda se encontra em um nível inaceitável. É raro que a forma clássica da doença acometa a classe média, mesmo em áreas endêmicas ${ }^{3}$. Segundo Bezzerra ${ }^{17}$, o grau de instrução dos pais é fator de proteção contra a infecção, o que vem corroborar o resultado da nossa pesquisa, na qual cerca de $80 \%$ das mães tinham apenas o ensino fundamental.

Comparando-se os casos comprovados e prováveis, constatou-se que não houve diferença estatística entre os dois grupos em relação a sexo, idade, procedência e estado nutricional. No entanto, quando avaliamos o tempo de evolução da doença, registramos que os casos prováveis tiveram um tempo de evolução significativamente mais curto (30,4 dias) do que os casos comprovados (45,9 dias).

A grande variabilidade em relação ao tempo de evolução da doença ( 2 dias a 1 ano) é compatível com os dados da literatura. Esta duração foi de 6 meses, em média, na experiência de Pastorino ${ }^{11}$ e de 1 a 5 meses na experiência de Marzochi ${ }^{18}$. O calazar é habitualmente uma doença insidiosa, com sintomas iniciais inespecíficos, fato que, aliado ao potencial viés de memória e ao baixo nível de escolaridade das populações nas áreas endêmicas, torna particularmente difícil a interpretação desta informação. No entanto, neste estudo, a alta proporção de casos (pelo menos $30 \%$ ) onde houve procura prévia por ajuda médica e uso prévio de outros medicamentos, como antibióticos, sugere que pode ter havido perda de oportunidade diagnóstica precoce, o que pode ser considerado preocupante em áreas onde a doença é endêmica, uma vez que o retardo no diagnóstico vem sendo implicado como um dos fatores de risco para óbito ${ }^{19}$.

A análise do estado nutricional mostrou a grande variabilidade clínica dessa doença, comprovada pela presença de pacientes com percentis normais para o peso (55\% das crianças), enquanto que aproximadamente $27 \%$ eram subnutridas graves. Observou-se que a maioria das crianças (83\%) tinha um tempo de evolução da doença inferior a 60 dias, tempo que pode não ter sido suficiente para o desenvolvimento das alterações nutricionais crônicas decorrentes da LV, o que poderia justificar a presença de pacientes eutróficos, como também foi observado por Pastorino ${ }^{11}$. É possível que a subnutrição possa suprimir a resposta imune mediada por células e ser responsável pelo desenvolvimento da leishmaniose visceral progressiva.

As manifestações clínicas do calazar nas crianças internadas são semelhantes àquelas descritas na literatura $^{1-3,5,7,11}$ tanto para os sintomas referidos pelos familiares 
quanto para os sinais físicos. Febre, hepatoesplenomegalia e emagrecimento são sinais clássicos da doença, presentes em quase todos os pacientes no momento da admissão, pois é durante este estágio que a maioria das pessoas chega ao ambulatório e ao hospital e se tem a oportunidade de firmar o diagnóstico. O fato de que muitas mães referiram uso prévio de medicação não é uma surpresa, em virtude dos sintomas iniciais serem comuns a outras doenças da infância, levando a uma confusão diagnóstica. A história de uso prévio da medicação específica para tratamento de calazar por $3,5 \%$ dos pacientes internados alerta para a possibilidade de falência ou refratariedade do tratamento, já descrita no Brasil anteriormente por Badaró 20 e que decorre, na maioria das vezes, de tratamentos inadequados.

A infecção é uma das principais complicações associadas à LV e é relatada inclusive nas formas subclínicas. Ocorre em indivíduos de todas as idades e, na forma clássica, associa-se a um curso fatal em cerca de $50 \%$ dos $\operatorname{casos}^{11,21,22}$. Nesta pesquisa, a infecção esteve presente em $10,9 \%$ dos pacientes no momento da internação, desenvolveu-se em $24,4 \%$ dos pacientes durante o internamento, estando associada ao calazar em $72,7 \%$ dos pacientes que foram a óbito, principalmente na forma de pneumonias, otites, infecções de pele e sepse. O encurtamento do tempo de permanência hospitalar e resultante diminuição da exposição dos pacientes a germes nosocomiais poderia ter influenciado na incidência de infecções hospitalares, o que deve ser mais bem explorado em estudos posteriores. Nesta casuística, vários fatores, isolados ou associados, merecendo destaque os valores médios de hemoglobinas, leucócitos e neutrófilos no início do diagnóstico, poderiam estar associados a infecções, como já destacado por outros autores ${ }^{20-22}$.

O envolvimento hepático tem sido relatado em 2 a $28 \%$ dos pacientes 23,24 , com maior freqüência quando o diagnóstico da LV é mais tardio e indica uma maior gravidade; no entanto, muitas vezes, tal envolvimento desaparece no curso do tratamento. As formas moderadas de hepatite são as mais freqüentes e, na grande maioria das vezes, diagnosticadas apenas laboratorialmente ${ }^{23}$. Em nossa pesquisa, $19(4,4 \%)$ crianças foram admitidas com icterícia. Existe a possibilidade de que o comprometimento hepático esteja subestimado, uma vez que as enzimas hepáticas não foram mensuradas de forma rotineira, sendo utilizado apenas o critério clínico (icterícia) para o diagnóstico de envolvimento hepático. O antimonial pentavalente (glucantime), droga utilizada para o tratamento da doença, tem a hepatoxicidade como um dos principais efeitos colaterais $7,21,25$ e pode contribuir para a falência hepática. Como computamos a icterícia apenas no momento da admissão, e nenhum desses pacientes tinha feito uso prévio recente de glucantime, o envolvimento hepático se deveu, provavelmente, à hepatite causada pela leishmânia, como observado anteriormente por Jerônimo em Natal ${ }^{25}$.

No presente estudo, fenômenos hemorrágicos estiveram presentes em $12,3 \%$ dos pacientes no momento da admissão e em cerca de $60 \%$ dos pacientes que foram a óbito, sendo um importante sinal de alerta de gravidade da doença.
Segundo a OMS, a anemia é registrada em $98 \%$ dos casos diagnosticados no Brasil e, quando grave $(<5 \mathrm{~g} / \mathrm{dl})$, é uma das indicações de internamento. Estudo prévio no IMIP 7 evidenciou que $88 \%$ dos pacientes eram anêmicos, enquanto que, na presente casuística, este fato ocorreu em $99,5 \%$ das crianças; em $25 \%$ dos casos, o nível de hemoglobina foi $<5 \mathrm{~g} / \mathrm{dl}$. É provável que a anemia seja de origem multifatorial, podendo decorrer de bloqueio de produção da medula, seqüestro esplênico, hemólise imune, hemorragia, parasitoses intestinais e carência de ferro. Destacamos que a anemia grave deve ser considerada um dos fatores mais importantes na vigilância e manuseio dos pacientes, inclusive com transfusão de hemácias, quando necessário. Leucopenia e neutropenia $\left(<1.500 / \mathrm{mm}^{3}\right.$ ) são encontradas com grande freqüência em pacientes com LV2,11,26, como verificamos em nossa pesquisa, na qual $85 \%$ das crianças evoluíram com leucopenia e $74 \%$ com neutropenia, provavelmente por hiperesplenismo, além de hipoplasia ou depressão medular e hemofagocitose. A plaquetopenia é um achado freqüente em pacientes com LV, presente em 50 a $70 \%$ dos pacientes ${ }^{11,26,27}$. Alves 7 , em pesquisa anterior no IMIP, encontrou plaquetopenia em $64,7 \%$ dos pacientes, achado semelhante aos deste estudo, nos quais $68,4 \%$ das crianças internadas tinham contagem de plaquetas inferior a $150.000 / \mathrm{mm}^{3}$. A contagem de plaquetas pode ser um fator preditor de hemorragia grave, que, em última instância, foi uma das causas imediatas de óbito, devendo, por isso, ter uma monitorização rigorosa.

Os resultados do presente estudo revelam uma taxa de letalidade de $10,2 \%$, que foi semelhante àquela encontrada em três hospitais de Natal (9\%) por Jerônimo ${ }^{25}$, em hospital-escola de São Paulo por Pastorino ${ }^{11}$ (9,3\%), assim como em estudo prévio no IMIP por Alves ${ }^{7}$. No entanto, esta taxa ainda pode ser considerada elevada quando comparada às taxas de letalidade nacionais, que variaram de $3,6 \%$ em 1997 a 6,3\% em 1990, segundo publicação do Ministério da Saúde ${ }^{14}$. A elevada letalidade encontrada neste e em outros estudos feitos em hospitais poderia ser decorrente do fato de que hospitais de referência e de maior complexidade poderiam atrair casos de maior gravidade potencial, selecionando uma clientela de maior risco de óbito. Além disso, os dados nacionais baseados em sistemas de notificação possivelmente subestimam o real número de óbitos decorrentes do calazar no país, em virtude do seguimento incompleto dos casos ${ }^{5}$. Em relação aos 44 óbitos registrados em nossa casuística, constatou-se que 38 eram crianças menores de 5 anos e 47,7\% eram subnutridos, achado compatível com outros dados da literatura7,19,24. As principais causas imediatas de óbito encontradas foram semeIhantes àquelas descritas por outros autores: infecção, hemorragia, anemia e insuficiência hepática 5,9,11,22,23. Apesar do conhecimento prévio das principais causas imediatas de óbito, estas persistem ao longo dos anos, embora haja evidência de descenso geral da letalidade ${ }^{6}$. Desta forma, ainda há indicação e espaço para uma vigorosa atuação objetivando uma redução significativa da letalidade, sendo importante que o agente de saúde, o profissional de enfermagem e o médico sejam capacitados para 0 reconhecimento e tratamento precoce da doença. Além 
disso, grande parte dos municípios ainda encontra dificuldades operacionais devido à deficiência da rede básica de saúde, em implantação, no que se refere ao diagnóstico, tratamento e notificação da LV, evidenciando uma maior necessidade de integração entre as ações de controle e de assistência médica para estes doentes.

\section{Referências}

1. Badaró R, Jones TC, Lourenço B. A Prospective study of visceral leishmaniasis in an endemic area of Brazil. J Infect Dis. 1986;154:639-49.

2. Berman JD. Human leishmaniasis: clinical, diagnostic and chemotherapeutic developments in the last 10 years. Clin Infect Dis. $1996 ; 24: 684-703$.

3. Ministério Nacional de Saúde. Fundação Nacional de Saúde (FUNASA). Controle, diagnóstico e tratamento da leishmaniose visceral (calazar): Normas Técnicas. Brasília; Ministério Nacional da Saúde; 1999. 85p.

4. Simplício ACR, Furtado JBV, Monteiro OS, Garret D. Leishmaniose visceral no brasil: análise epidemiológica nos últimos 16 anos. Rev Soc Bras Med Trop. 2002;35:298.

5. Correia JB. Epidemiology of visceral leishmaniasis in Pernambuco, north-east of Brazil and the use of a latex agglutination test in urine for its diagnosis [dissertation]. Liverpool: Liverpool School of Tropical Medicine; 1998.

6. Vieira JBF, Simplício ACR, Monteiro PS. A letalidade por leishmaniose visceral no Brasil. Rev Soc Bras Med Trop. 2002;35:322.

7. Alves JGB. Calazar. In: Figueira F, Ferreira OS, Alves JGB. Pediatria - Instituto Materno infantil de Pernambuco. $2^{a}$ ed. Rio de Janeiro: Medsi; 1996. p. 320-27.

8. Arias JR, Monteiro OS, Zicker F. The reemergence of visceral leishmaniasis in Brazil. Emerg Infect Dis. 1996;2(2):145-6.

9. Organização Pan Americana de Saúde (OPAS)/Organização Mundial de Saúde (OMS). Manual de Controle da Leishmaniose Visceral. Brasília; 1997. 89p.

10. Berman J. Miltefosine, an oral agent for the treatment of India visceral leishmaniasis. N Engl J Med. 1999;341(24):1795-800.

11. Campos Jr D. Características do calazar na criança. Estudo de 75 casos. Pediatr (Rio J). 1995;71:261-5

12. Pastorino AC, Jacob CMA, Oselka GW, Sampaio MMC. Leishmaniose visceral: aspectos clínicos e laboratoriais. J Pediatr (Rio J). 2002; 78:120-7.

13. Costa HNC, Pereira HF, Araújo MV. Epidemia de leishmaniose visceral no estado do Piauí, Brasil 1980-1986. Rev Saúde Pública. 1990;24(5):361-72.

14. Ministério da Saúde. Fundação Nacional de Saúde. Boletim epidemiológico. Evolução Temporal das Doenças de Notificação Compulsória no Brasil de 1980 a 1998. Brasília: CENEPI/ FUNASA; 1999
15. Silva AO, Silva PB, Silva OV, Melo AA, Leite JA, Pinheiro AJ, et al. Leishmaniose visceral no agreste Pernambucano: casos humanos. Rev Bras Med Trop. 2001;34 Supl:221.

16. Andrade PP. A situação das leishmanioses em Pernambuco. Consulta para o Governo do Estado de Pernambuco; 1996. 46p.

17. Bezerra GFB, Souza EC, Silva MH, Bandeira Neto AP, Barbosa R, Campelo FCC, et al. Estudo dos fatores de risco por anticorpos anti-leishmania em área endêmica de leishmaniose visceral na Ilha de São Luís - MA. Rev Soc Bras Med Trop. 2002; 35 Supl:310.

18. Marzochi MCA, Sabroza PG, Toledo LM, Marzochi KB, Tramontano NC, Rangel Filho FB. Leishmaniose visceral no Rio de Janeiro Brasil. Cad Saúde Pública. 1985;I(1):5-17.

19. Seaman J, Mercer AJ, Sondorp HE, Herwaldt BL. Epidemic visceral leishmaniasis in Southern Sudan: treatment of severely patients under wartimate conditions and with limited resources. Ann Intern Med. 1996;124:664-72.

20. Badaró R, Duarte MI, Luz KG. Leishmaniose visceral. In: Farhat CK, Carvalho LM, Succi RC. Infectologia Pediátrica. $2^{a}$ ed. São Paulo: Atheneu; 1998. p. 563-78.

21. Andrade TM, Carvalho EM, Rocha H. Bacterial infectious in patients with visceral leishmaniasis. J Infect Dis. 1990;162: 1354-9.

22. Guerreiro J, Ribeiro S, Carvalho DM, Badaró R, Rocha $H$. Infecção bacteriana em pacientes portadores de leishmaniose visceral. Memórias do Instituto Oswaldo Cruz, Rio de Janeiro. 1985;80:447-52.

23. Khaldi F, Bennaceur H, Othoman BH, Achouri E, Ayachi R, Regaig $R$. Les formes sévères d'atteinte hépatique au cours de la leishmaniose viscérale. Arch Fr Pediatr. 1990;47:257-60.

24. Harrat Z, Berrouane $Y$, Abdesslam SB, Belkaid M, Tabet-Derraz O. La leishmaniose viscerale em Algerie evolution de la leishmaniose viscerale dans le Foyer de Grande Kabyle. Arch Inst Pasteur Algérie. 1992;58:255-72.

25. Jerônimo SMB, Oliveira RM, Mackay S, Costa RM, Sweet J, Nascimento ET, et al. An urban outbreak of visceral leishmaniasis in Natal, Brazil. Trans R Soc Trop Med Hyg. 1994;88:386-8.

26. Nasir AM, Al-Nasser MN, Al-Jurayyan NAM, Al-Fawaz IM, AlAyed IH, Al-Herbish AS, et al. The haematological manifestations of visceral leishmaniasis in infancy and childhood. J Trop Pediatr. 1995;41:143-8.

27. Elnour IB, Akinbami FO, Shakeel A, Venugopalan P. Visceral leishmaniasis in Omani children: a review. Ann Trop Paediatr. 2001;21:159-63

Correspondência:

Márcia Jaqueline Alves de Queiroz

Rua Januário Barbosa, 160/201

CEP 50610-060 - Recife, PE

Tel.: (81) 3227.5625 - Fax: (81) 3228.1215

E-mail:m_msampaio@uol.com.br 